\title{
Innovative technologies of wireless sensor network: The applications of WBAN system and environment
}

\author{
Israa Al Barazanchi ${ }^{1}$, Haider Rasheed Abdulshaheed ${ }^{2}$, Madya Safiah Binti Sidek ${ }^{3}$ \\ ${ }^{1,2}$ Baghdad College of Economic Sciences University - Baghdad - Iraq, \\ ${ }^{1,2}$ Faculty of information and communication technology, Universiti Teknikal Malaysia Melaka, Melaka, Malaysia \\ ${ }^{3}$ Institut Pengurusan Teknologi \& Keusahawanan (IPTK), Universiti Teknikal Malaysia Melaka (UTeM), Melaka, Malaysia
}

*Corresponding author: israa44444@gmail.com

\section{(C) The Author} 2019.

Published by

ARDA.

\begin{abstract}
Wireless Body Area Networks (WBAN) have risen as a key innovation for providing real-time patient health care and diagnosing many life-threatening diseases. The principle adopted by health service providers is providing convenience for patients in need of health care, allowing them comfortable with the movement and constant monitoring with a reduction in health care costs. This study presented the basic concept and general background of the main WBAN system with applications. The current models for on-body and embedded WBANs, sensor node features, and sensor schemes for information via negation are used in the WBAN.
\end{abstract}

Keywords: WBAN, Health monitoring, Communication technologies, Environment

\section{Introduction}

WBAN refers to a group of small intelligent electronic devices placed on the human body to monitor its vital signals. It covers the requirements of continuous health monitoring of the patient without any restriction on the activities of his ordinary everyday life through health care applications. Because of the solid heterogeneous nature of the applications, information rates will differ emphatically, running from straightforward information at a couple of Kbits/s to video stream of a few Kbits/s. Information can likewise be sent in blasts, which implies that it is sent at a higher information rate during the blasts. Because of that, the proficiency of system execution in social insurance applications is one of the most significant essentials for structuring a compelling reconnaissance framework [1].

In other words, the WBAN system can be defined as a set of wired or wireless electronic devices that come together to build a system that continuously monitors the patient's vital signals through the sensors on his body. There are different types of wave transfer modes adopted in WBAN systems, in this thesis explain the main characteristics of these types and clarify the type of difference between them, where only one of the media recommended by the researcher is highlighted based on the efficiency shown in several previous studies. Figure 1 show the main system of wireless body area network.

Based on Figure 1, the main component of WBAN systems are sensors, CCU, and receiver devices. The section 3 explains in detail the specification of the main components for WBAN system.

This work is licensed under a Creative Commons Attribution License (https://creativecommons.org/licenses/by/4.0/ ) that allows others to share and adapt the material for any purpose (even commercially), in any medium with an acknowledgement of the work's authorship and initial publication in this journal. 


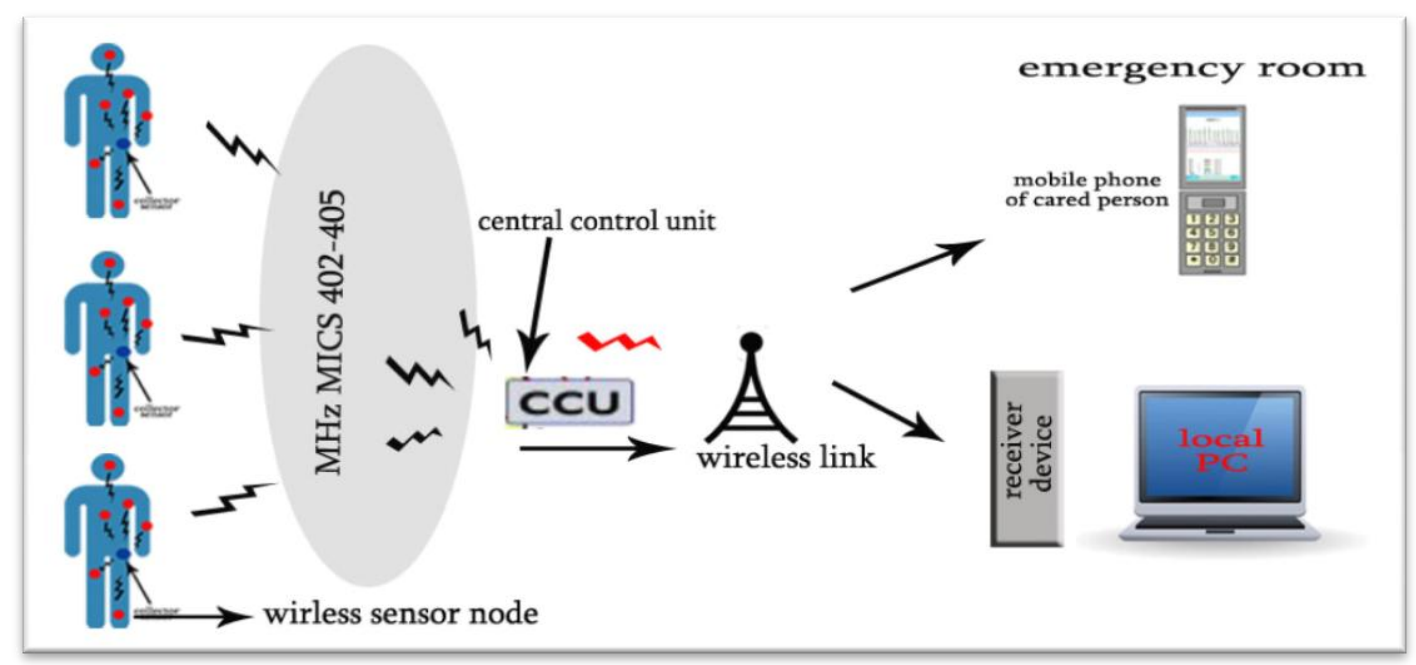

Figure 1. The architecture of wireless body area network

\section{WBAN applications}

The development use of WSN accompanying the continuous reduction of electronic devices has led to the development of networks of WBAN [2]. WBAN provides remote monitoring services without any restrictions and on a continuous basis with the possibility of doing daily activities. Networks and competencies have been proven in several applications. The most used applications of the WBAN technology in the health care area are listed below [3].

\section{- Athlete monitoring}

The WBAN techniques were utilized to measure the movements of athletes accurately in order to improve the performance of athletes to suit the requirements of the field of modern sports. Since the vital indicators rely upon the athletic performance, it is impossible for the athletes to be interlinked with wires to the delivery center or a specific destination in order to follow their indicators. Therefore, the new WBAN technologies allow monitoring of the vital indicators by the installation of sensor devices in different places and in ways that suit their movement and are broadcast wirelessly to the concerned party.

\section{- Soldier surveillance}

The WBAN technology made its way into the battlefields through the monitoring of the wounded and sick by following up their vital indicators. This is to ensure proper treatment of the soldiers as well as to determine those who must be sent to specialized medical centers. In addition, the WBAN technology allows early detection of explosives through temperature and noise sensors, which could save the lives of many soldiers. Other cases involving soldiers in the battlefield are the detection of soldiers who suffered from tachycardia or sudden exposure to extreme heat at bedtime, which may be the cause of many deaths.

\section{- Advanced animal care}

The sensor technology makes the monitoring of endangered animal species possible. This situation allows animal specialists to keep track of the animals' health condition whereby the sensors are implanted within the fabric attached to the body of the animals. The movement of the animals can be monitored, while other variables such as temperature can be recorded, without causing discomfort to the animals, as they will behave in a more natural movement.

\section{WBAN system}

WBAN framework allude to the principle part or components of a patient checking framework in a healthy environment. A WBAN structure licenses basic internetworking with various contraptions and frameworks, thusly offering therapeutic administrations pro straightforward access to the patient's fundamental and nonessential data. One of the essential inclinations of a WBAN is to screen patients remotely using an intranet or 
the Internet. The WBAN framework comprises of two primary parts, programming and equipment [7]. The product depends on the getting gadgets, which is chosen by the provided organization. The equipment comprises essentially of comprises of three primary components that incorporates sensor node, a CCU that transmits information to a nearby personal computer lastly, a getting station at a restorative focus. Crude information from a patient body is removed by sensing node [8]. The principle equipment part in WBAN framework comprises of the accompanying gadgets:

\subsection{Sensor nodes in WBAN system}

Sensor node is designed to collect vital data from the human body. The specification of the sensor node in WBAN system are low-power average, size, and suitably operating in the special type band like the MICS for physiological data processing. in almost WBAN system the sensors node contains same hardware components; the sensors collect vital patient data. Normally the signal collected from the human body is not strong and usually accompanied by noise due to different sound inside the body like the heart pulse.

At the point when the sensor gets a sign information, it enhances them to make them more grounded, which was then separated to expel undesirable seems like clamor so as to set it up to experience an (ADC) stage to be changed over the computerized structure in advanced handling. The digitized sign is then handled and put away in the microchip [9]. The sensors give a consistent wellbeing checking of a patient with no imperative on his/her typical day by day life exercises through the human services applications. Every sensor hub contains the accompanying equipment parts. The parts are:

- Sensing unit: movement sensor (3- axis accelerometer) and temp sensor (NTC Thermistor).

- Power supply unit: $3 \mathrm{~V}$ coin-cell Lithium battery.

- Micro Controller Unit (MCU): Texas Instruments (TI's) MSP430 microcontroller.

- Communication unit: ZigBee-good radio handset dependent on TI CC2480 chipcon.

- Memory/Storage unit: 1Kbyte on-chip RAM memory, and 32Kbyte on-chip FLASH.

The estimation of electric essential sensors and their resulting handling for include extraction, prompts an accumulation of constant physiological parameters assembled, which can give a general estimation of the client's wellbeing condition at some random time as expressed by[4]. The table shows different types of sensor nodes used in the WBAN system with details of where they are placed (in which part of the body) and the function they perform. These types are the most commonly used in the monitor of patient vital data, there are cases to use only one or two types to monitor certain indicators. To design any monitoring system, its important to focused on the quality of the sensors manufacturer and the specifications of the chip and ensure that all the sensors are compatible with each other to ensure receiver devices can receiving patient signals without problems in time of send and receive.

\subsection{CCU in WBAN system}

For any WBAN system, the CCU is one of the important component systems, which gathers all the transmitted information from sensors in the WBAN and processes the data for monitoring or diagnostic purposes. Data is moved from the RF associate with the CCU through the wave like MICS band once taking care of has been done. Next, the information is re-bundled by the CCU before being transmitted to the nearby PC. In cases, where remedial centers place at another zone, data from the local PC is transmitted to a remote PC by methods for web or any framework in the therapeutic core interest. Bluetooth Communication is partnering the CCU to PDA, goes about as a consequent remote association, which persuades future work. This suggests, of correspondence is as yet a work in progress. This remote association improves patient's convey ability level, since they can go out to perform step by step practices [8]. Generally, all information transfer directly to the recipient station (for example the personal computer) can be appeared on a User Display Graphic (GUI). The beneficiary station can in like manner store every one of the information found in the database plan of a helpful core interest. CCU is planned to be worn around a patient's stomach region, also 
depending upon understanding adaptability. Patients holding quick to confined improvement, will have the CCU set at sections in their homes, inside a detachment of 10 meters. The equivalent the employments of a remote PC at the restorative center, the CCU at home, is related with a local PC where predictable data which is gotten from the sensor hub, other than being register to a secretly set database. Data which is dealt with can be passed on to the database of the restorative center enthusiasm, by methods for the web. This should be conceivable irregularly. In conditions where there is more than one patient living in a room (Ex. Crisis center), key programming groups are presented in the CCU and the close by PC. This is done to change physiological sign from sensors of each patient independently. Information from patients are gathered in comprehension to time breaks that are consigned to patients. Two basic pointers that are picked to screen model structure and to show a multi customer framework are patients' heartbeat rate and temperature [12].

\section{WBAN system organization}

The WBAN is usually organized / positioned on or near the human body, where sensors are attached as a fixed base to a frame or wrist. The sensors are designed with the ultimate goal of collecting vital signs from the human body, because the mark of the human body is usually weak and accompanied by noise. The signal must face an intensification and separation process towards starting to expand the signal qualities, getting rid of unwanted flags and noise. The location of the nodes on the body and more specifically the path to the other nodes has a significant impact on the quality of the channel. As such, a slightly different situation can lead to a different channel, because the visible line of communication suddenly becomes impossible. This means that different people will also have a different channel quality, as the shape of the body also affects the channel [5]. Because of this highly situation-specific channel, this work is not targeted to pre-compute or determine the best location of the sensors. Instead, random node locations with approximate possible WBAN node locations will be generated. This work wants to focus on the implications of the given channel, rather than trying to optimize the channel itself. The quality of WBAN channels is a major challenge for networks, as they will limit possible solutions. One interesting opportunity is the small scale of the network. Patients probably don't have hundreds of sensors attached to their body. Application of the WBAN network in a medical environment may include wearable and implantable sensor nodes that distinguish biological information about the human body. Besides, it also transmits information over a short distance wirelessly to a controller, worn on the body or placed in an accessible place. Sensor electronics should be miniature and lowpower. In addition, it should be able to sense medical signals such as ECG, PPG, EEG, pulse rate, pressure and temperature [6]. The collected data was then transferred from the controllers to remote destinations. This is done via WBAN, which is intended for diagnostic and therapeutic purposes. Besides, it also allows the integration of another wireless network for long range transmission as shown in Fig. 4. Restorative focuses right now use observing gadgets that are not totally wearable because of hardware that are massive and wires that are utilized for interfacing numerous sensors. Figure 4 delineates the use of a sensor organize that is as of now utilized in a specific best in class therapeutic focuses. CCU collects data from sensors via wires before transferring them to a remote device for monitoring purposes. The depending of wireless is not enabled in this procedure because it carries discomfort for patients. In addition, the console can also be unmanageable. Future therapeutic sensing systems require a sensor node that is minimized and wearable and can be discussed remotely using the information acquisition tool. An individual's physiological data, for example, beat, rotational strain and ECG can be transmitted through a remote association, which comprises of an individual remote detecting hub. This should be possible without sending any wire affiliation. Another reality that must be incorporated is that every sensor has its very own remote ability and its structure will be created regarding the physical attributes of the physiological marker [7]. Individual nodes are one or more points, where some patients only need all the physiological parameters for analysis. In future medical service conditions, adaptive innovation and rubber can be used to plan nodes that can be inserted into patient attire, to improve patient comfort. Two basic physical necessities of the sensor node are low energy activity and downsizing. These variables determine the age of the instruments just as they are suitable for patients. 


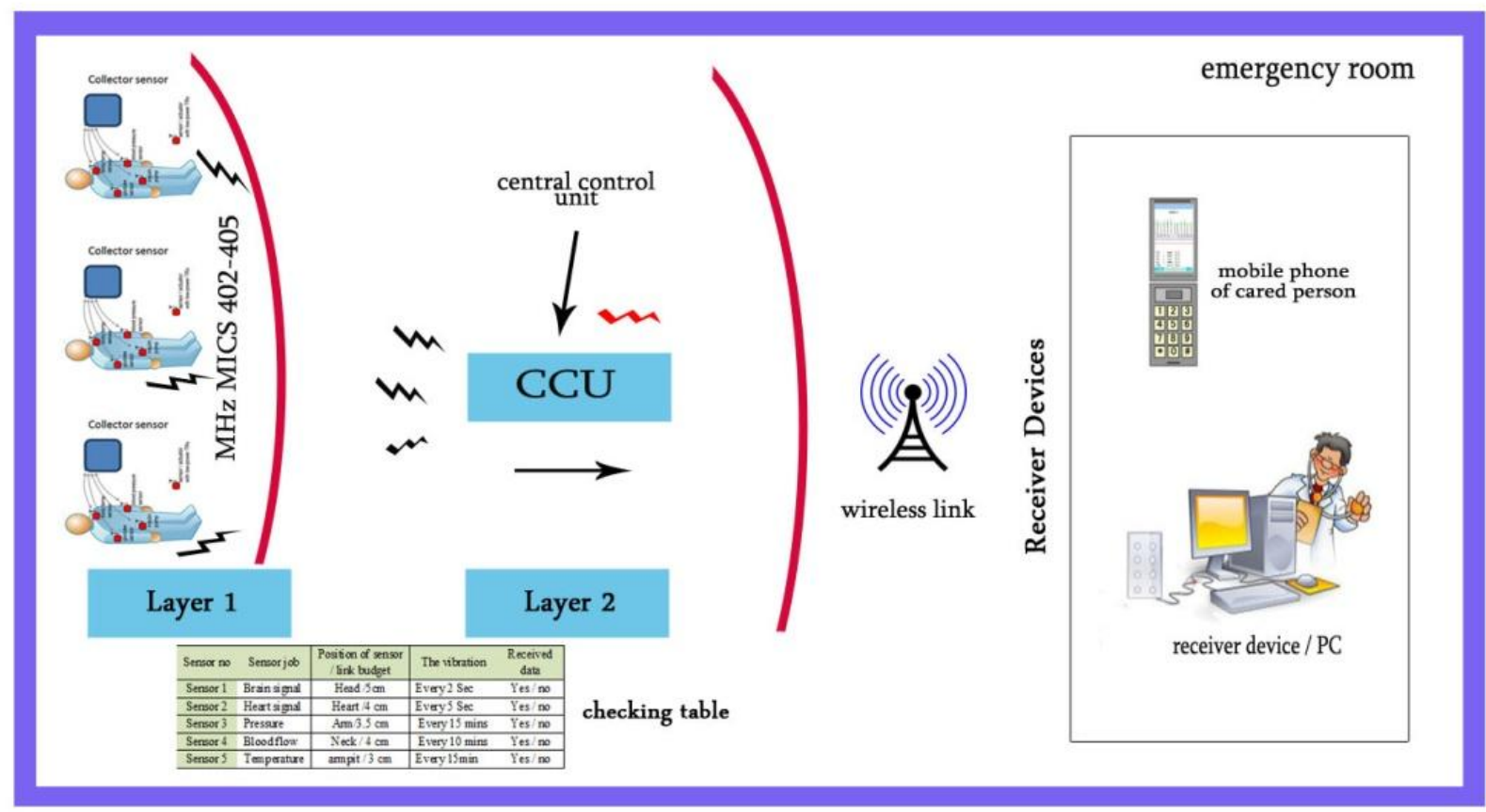

Figure 4: WBAN system that detects and transmits signals from a human body.

Remote chip activity and radio transmission and frequency (RF) are controlled using energy to hold the sensor. Thus, the far-off phase, which provides low-power use with the lowest capacity of transmitters, is best as it can meet the range needed to arrange the body area.

\subsection{Example of WBAN system}

The wireless body sensor network consists of three basic components including the sensor node, the CCU that transmits information to a nearby computer soon, and is a therapeutic concentration acceptance tool. The raw information is cleared from a human body by the sensor node. The information is transmitted from the wireless connection to the CCU through the Medical Implant Communications Service (MICS) once the preparation has been completed. The information is then regrouped by the CCU before it is sent to the nearby PC. In cases where there are therapeutic focus points in different areas, information is transferred from the nearby PC to a remote computer via the Internet or any system in restorative focus. [8] Future work. This means that the correspondence is still in progress. This remote connection improves the patient's susceptibility, as they can go out for daily exercises. Each of the information obtained by the patron tool (for example the remote computer) can be displayed on the graphical user interface (GUI) stage. The wrapper tool can also store all the data in the database order of the therapeutic concentration. The CCU is intended to be worn around the patient's heart, which depends on the patient's portability, and patients who stick to restricted development will place the CCU in corridors in their homes, within a 10-meter classroom. The same functionality of the remote computer in restorative focus, CCU at home is connected to a nearby computer where the continuous data obtained from the sensor node is displayed, other than recorded in a private database. The data that is placed away to the medical focus database can be transferred via the Internet, which should sometimes be possible. In situations where more than one patient lives in a room (e.g. a restorative center), essential programming bundles are offered in the CCU and the close by PC. This is done to change the physiological marker of the sensor for every individual patient. Information from patients are assembled in concurrence with time intervals that are moved to patients [9].

\subsection{Sensors communication in the human body}

In today's globalized society, developments in the field of electronics, telecommunications and specifically integrated circuit development in enhanced surveillance sensors are necessary for the ease of movement and to 
monitor vital signs. These electronic devices play an important role in collecting physiological information from a patient's body and sending it to the medical center so that the data is not lost, but rather arrives at the medical center in a secure and reliable way.

Hence WBAN has become a modern trend in the development of electronic circuits and protocols. To add, since the well-being of a patient as well as follow ups of their health issues have always been the aim of doctors and medical staff, it is crucial to conduct studies to identify the problems faced by sensors within the human body [10]. Wireless networks can serve as a communication medium with in-body nodes, e.g. pacemakers. Because of the very specific channel inside the human body and the very high demands with respect to energy efficiency, this work limits itself to on-body networks. As illustrated in Figure 5, a WBAN consists of multiple nodes located in different locations on the human body, A Number of devices are Attached to the Joints and Limbs, While Sensor D Measures the Heart Rate. The area of a sensor essentially relies upon the capacity of the sensor appended to the node. Therefore, depending on the required parameters to monitor, different sensors and different network topologies will arise.

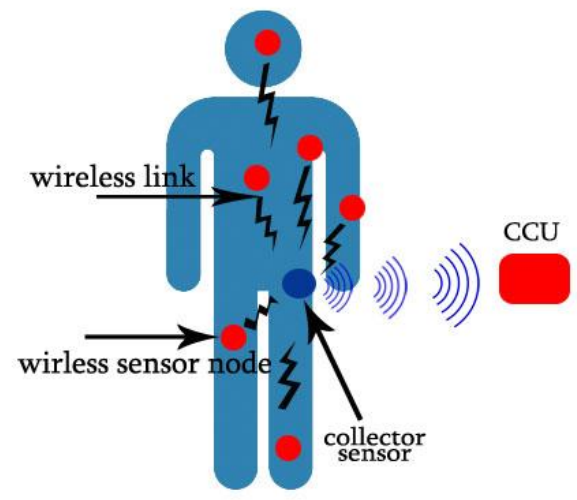

Figure 5: Schematic of an example WBAN on the human body

Body Sensor Network (BSN) will be used to refer to the network as a special case of Wireless Sensor Network (WSN), where the sensors now gather data on a very small scale as opposed to the classical large scale WSNs. Moreover, there seems to be a trend towards defining WBAN research focused on the communication network while BSN research will focus on processing the data obtained by means of a WBAN. The IEEE defines one other network type near the body as the Wireless Personal Area Network (WPAN). The main difference between a WBAN and a WPAN lies in the data rates, whereby WPANs will use technologies like Bluetooth to support sustained rates of up to $3 \mathrm{Mbps}$. This is significantly higher than the envisioned rates from the sensor on the human body. Therefore, the WPAN network will be formed in the personal operating space of about $10 \mathrm{~m}$ around the body, e.g. between a smart phone and a printer, while a WBAN will be formed in the body [11].

\subsection{Patient monitoring status via hospital devices}

Usually, when the patient enters the hospital, the medical team questions him about his indications. When the answer indicates heart disease or those relating to the blood vessels, the patient will have to stay in the hospital for a certain period of time depending on the tests done upon him. The survival of the patient in the hospital is taken as an indicator of successfully linked sensors to monitor vital indicators within the system to the doctor and the center of monitoring patients. Modern systems are moving towards the use of medical devices connected to each other wirelessly while ensuring efficient signal transmission and minimizing the impact of air humidity and interference of other waves in place considering the location of the patient for the receiving devices. There is a need to develop solutions to address the problem of packet loss during the transmission based on the design of a system that ensures transmission of patient's data in the monitoring room and outside without causing change or loss, taking into account the patient's comfort and the distance away from the receiving devices [12]. 


\subsection{WBAN topologies}

Generally, WBANs contain numerous sensor nodes that spread throughout the patient's body area and each WBAN explicitly has one CCU. The sensor and CCU nodes are organized in one of the system topologies [13]:

Star Network

Between node correspondence does not exist between the sensor node and the local node; paths are either oneway or two-way.

Hyperlinks

The sensor node contains two-way correspondence with another sensor node and a local node. Whatever the case, the remote sensor node communicates with the CCU but not with another sensor node, when inside a star arranging the design. The sensor node continues to run on restricted power and account assets. In this way, some WBANs use one-way correspondence between the sensor node and the CCU to protect battery life. In the meantime, in the work order design, a larger power source is required at the end of the sensor panel instead, as the sensor node moves to the non-CCU. For stellar systems, the minimum sign preparation occurs on the node and the raw signal delivered from the sensors is changed to an advanced arrangement before continuing the transmission box. In general, this aims to reduce the use of control, as it reduces the computational complexity of the sensor node. The movable raw mark is then passed and collapses in the CCU, where the energy reduction is less. The input channel from the CCU to the sensor node does not exist, so the synchronization or time reservation methods are unrealistic due to the energy-related nature of the sensor node. In this way, an abnormal WBAN is created where the sensor node transmits information to the CCU at predefined intervals that are ambiguous for a different node in the system [14].

\section{Conclusion}

In this study, an explanation was given to the meaning of the network of sensors to monitor the vital signs of the patient's body with an explanation of the types of these systems, in addition to a detailed explanation of the nature of the sensor work and how to connect it with the network. In this chapter presented the basic concept and general background of WBAN, WBAN system, communication in WBAN and give approach for the network performance in WBAN.

\section{References}

[1] I. I. Sadiq, "IDENTIFICATION KEY SCHEME TO ENHANCE NETWORK PERFORMANCE IN WIRELESS BODY AREA NETWORK," UNIVERSITI TEKNIKAL MALAYSIA MELAKA, 2019.

[2] I. Al Barazanchi, A. S. Shibghatullah, and S. R. Selamat, "A New Routing Protocols for Reducing Path Loss in Wireless Body Area Network (WBAN )," J. Telecommun. Electron. Comput. Eng. Model, vol. 9, no. 1, pp. 1-5, 2017.

[3] B. Braem, "Reliable and Energy Efficient Protocols for Wireless Body Area Networks," Universiteit Antwerpen (Belgium). [Online]. Available: https://dl.acm.org/citation.cfm?id=2395363.pdf. accessed: 12-Feb.-2016], 2011.

[4] A. Pantelopoulos, "Prognosis: a Wearable System for Health Monitoring of People At Risk," Electrical and Computer Engineering, University of Patras. [Online]. Available:https://etd.ohiolink.edu/rws_etd/document/get/wright1284754643/inline.[accessed: 16-May2016]., 2010.

[5] B. Braem and C. Blondia, "Supporting mobility in Wireless Body Area Networks: An analysis," Commun. Veh. Technol. Benelux (SCVT), 2011 18th IEEE Symp., pp. 1-6, 2011.

[6] V. Goverdovsky, D. Looney, P. Kidmose, C. Papavassiliou, and D. P. Mandic, "Co-Located Multimodal Sensing: A Next Generation Solution for Wearable Health,” IEEE Sens. J., vol. 15, no. 1, 
pp. 138-145, 2015.

[7] I. Al Barazanchi, H. R. Abdulshaheed, and A. Shibghatullah, "The Communication Technologies in WBAN," Int. J. Adv. Sci. Technol., vol. 28, no. 8, pp. 543-549, 2019.

[8] A. S. Shibghatullah and I. Al Barazanchi, "An analysis of the requirements for efficient protocols in WBAN," J. Telecommun. Electron. Comput. Eng., vol. 6, no. 2, 2014.

[9] I. Al Barazanchi, H. R. Abdulshaheed, S. A. Shawkat, and S. R. Binti, "Identification key scheme to enhance network performance in wireless body area network," Period. Eng. Nat. Sci., vol. 7, no. 2, pp. 895-906, 2019.

[10] S. Ullah, P. Khan, N. Ullah, S. Saleem, H. Higgins, and K. S. Kwak, “A Review of Wireless Body Area Networks for Medical Applications,” arXiv Prepr. arXiv1001.0831, p. 7, 2010.

[11] T. Dou and L. Yi, "The Development and Trend of Wireless Body Area Networks in The Smart Clothing Field," 4th Int. Conf. Mechatronics, Mater. Chem. Comput. Eng., no. Icmmcce, pp. 1182 1187, 2015.

[12] Liao et al., "Analysis of In-to-Out Wireless Body Area Network Systems : Towards QoS-Aware Health Internet of Things Applications," Electronics, vol. 5, no. 3, p. 38, 2016.

[13] A. Shibghatullah and I. Al Barazanchi, "A survey on Central Control Unit ( CCU ) in WBAN," Int. Symp. Res. Innov. Sustain. 2014 (ISoRIS '14) 15-16 Oct. 2014, Malacca, Malaysia, vol. 14, no. October, pp. 15-16, 2014.

[14] E. Iliana and R. Carlos, "WBAN oriented for Smart Health WBAN orientado para Smart Health," ECORFAN-Bolivia J., vol. 5, no. 9, pp. 43-48, 2018. 